\title{
Review
}

Peter Schuff-Werner*, Joe Mansour and Angela Gropp

\section{Pseudo-thrombocytopenia (PTCP). A challenge in the daily laboratory routine?}

https://doi.org/10.1515/labmed-2020-0099

Received August 25, 2020; accepted August 26, 2020; published online September 17, 2020

\begin{abstract}
Anticoagulation with ethylenediaminetetraacetic acid (EDTA) is a necessary pre-requisite for automated blood cell counting. With a prevalence of $0.01-1 \%$, EDTA anticoagulation is accompanied by time- and temperature-dependent in vitro aggregation of platelets, resulting in false low counts. To avoid wrong clinical conclusions, spontaneous anticoagulant-induced platelet agglutination should therefore be recognized during analysis. This might be a challenge for the routine laboratory.

The actual knowledge of this rare but clinically important laboratory artefact will be summarized and reviewed in the following, based on our own experiences and the available literature. This includes pathophysiological and epidemiological aspects, valuable information regarding the detection and prevention of a PTCP, and the possibilities for determination of the correct platelet count.
\end{abstract}

Keywords: analyzer flagging; anticoagulant-induced pseudo-thrombocytopenia (PTCP); misleading diagnoses; PTCP prevalence; PTCP recognition strategies.

\section{EDTA-dependent pseudothrombocytopenia (PTCP)}

Complete blood cell count (CBC) is the most commonly ordered analysis in the routine hematology laboratory. In the early 1950s, hematologists recommended ethylenediaminetetraacetic acid (EDTA) as the most suitable

\footnotetext{
*Corresponding author: Prof. em. Dr. med. Peter Schuff-Werner, Universitatsmedizin Rostock, Institute of Clinical Chemistry and Laboratory Medicine, Ernst-Heydemann-Str. 6, Rostock, Germany, E-mail: pschuffw@med.uni-rostock.de

Joe Mansour and Dr. med. Angela Gropp, MDI Labor Limbach Berlin (Medical Care Center), Berlin, Germany, E-mail: joe.mansour61@gmail.com (J. Mansour), angela.gropp@mvz-labor-berlin.de (A. Gropp)
}

anticoagulant for cell counting and WBC differentiation [1] which later became the standard anti-coagulant with the introduction of automated cell counters. The irreversible calcium-complexing capacity of EDTA prevents clotting and therefore stabilizes the fluidity of whole blood samples for electronic particle counting. Another advantage of EDTA-anti-coagulation are the good and stable staining properties for white blood cells [2]. However, time dependent cell swelling and rare but non-negligible spurious thrombocytopenia (pseudo-thrombocytopenia) are disadvantages of EDTA anti-coagulation, first reported by Gowland in 1969 [3]. The anticoagulant-related false low platelet count results from spontaneous aggregate formation in vitro (Figure 1). This phenomenon is therefore a laboratory diagnosis of EDTA incompatibility and not a clinical diagnosis.

The present review gives an overview on the published knowledge of pseudothrombocytopenia (PTCP) including its pathophysiology and epidemiological and clinical data. We will also present experiences from the laboratory on how to recognize and so avoid false low platelet count reports to clinicians.

\section{PTCP as a source of misleading clinical conclusions}

Although PTCP was quickly recognized as a preanalytical problem, alleged low platelet counts reported by the laboratory provided an important confounding factor in the management of patients, leading to wrong decisions and diagnoses [4]. Within the last five decades, multiple cases of misdiagnoses or mismanaged patients due to spurious low platelet counts were communicated; most were of minor medical impact, but others were significant. Four decades after the first reports of PTCP, the cardiologists of a university hospital intended to avoid performing a necessary invasive examination because of a supposedly low platelet count. Fortunately, a laboratory technician noticed the suspicious histogram and proposed to repeat the platelet count by using another anticoagulant [5]. 


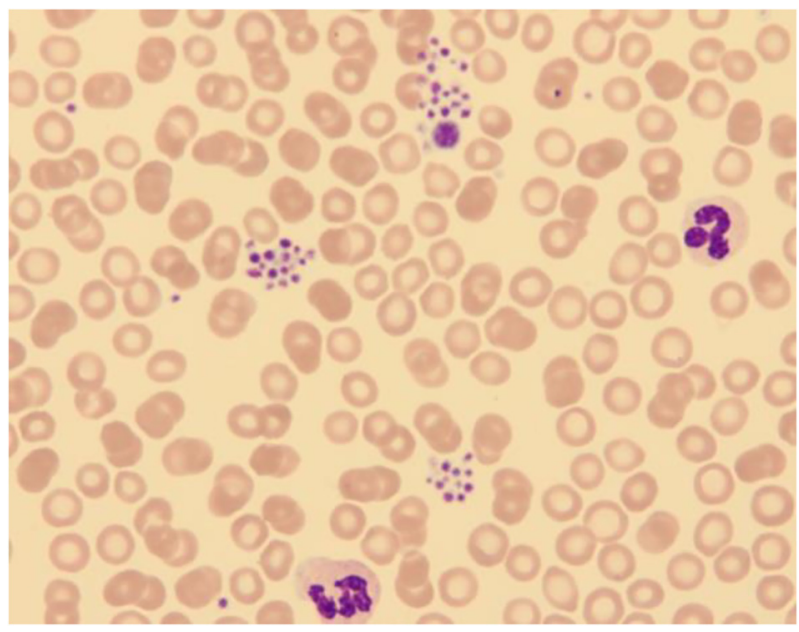

Figure 1: Large platelet aggregates and a giant platelet in the peripheral smear of EDTA-anticoagulated blood.

Table 1 summarizes some typical examples of misinterpretation and wrong medical decisions reported in the respective literature. The most communicated incorrect diagnosis was the suspicion that the prescribed drug led to falling platelet counts, leading to discontinuation of the regimen [6]. For example, if clinicians suspected heparininduced thrombocytopenia (HIT) in systemically anticoagulated patients, they stopped heparin therapy [7].Another example includes an assumption that ITP was responsible for the marked thrombocytopenia, and so PTCP-patients were regularly but unnecessarily treated with high doses of corticosteroids [4, 8]. Finally, in supposed therapy-resistant ITP, treating physicians provided an indication for splenectomy $[9,10]$.

\section{Pathophysiological considerations}

Under physiological conditions, platelets aggregate when activated, such as by taking blood after venous or arterial vein congestion. A proper blood sampling technique in appropriate anticoagulated tubes and a careful mixing of the sample is necessary to avoid platelet aggregation in vitro [16]. A rather rare but improper technique to get blood for platelet count is to transfer blood accidentally drawn without anticoagulant to a collection tube anticoagulated with EDTA. Polystyrene beads from a serum separation tube in the EDTA tube randomly revealed such a preanalytical misconduct (own observation).

EDTA irreversibly complexes calcium, magnesium, and other divalent cations. As an alternative anticoagulant, sodium citrate reversibly binds calcium ions, which are essential for the coagulation process. In laboratory
Table 1: Selected examples for misleading diagnoses and false therapeutic decisions because of alleged thrombocytopenia due to unidentified pseudothrombocytopenia (PTCP).

\begin{tabular}{|c|c|c|}
\hline PTCP-related misdiagnosis & $\begin{array}{l}\text { Misguided therapeutic } \\
\text { decision }\end{array}$ & Reference \\
\hline \multirow[t]{3}{*}{$\begin{array}{l}\text { Thrombocytopenia of un- } \\
\text { known cause }\end{array}$} & $\begin{array}{l}\text { Failure to implement a } \\
\text { planned action of inva- } \\
\text { sive cardiological } \\
\text { intervention }\end{array}$ & {$[5,11]$} \\
\hline & $\begin{array}{l}\text { Retardation of anti- } \\
\text { coagulation and/or } \\
\text { fibrinolysis in acute MI } \\
\text { or stroke }\end{array}$ & [12] \\
\hline & $\begin{array}{l}\text { Unnecessary transfusion } \\
\text { of platelets }\end{array}$ & [13] \\
\hline $\begin{array}{l}\text { Antiviral treatment induced } \\
\text { thrombocytopenia in virus } \\
\text { infection }\end{array}$ & $\begin{array}{l}\text { Cessation of antiviral/an- } \\
\text { tiretroviral medication }\end{array}$ & [6] \\
\hline $\begin{array}{l}\text { Drug-induced } \\
\text { thrombocytopenia }\end{array}$ & $\begin{array}{l}\text { Discontinuation of the } \\
\text { drug }\end{array}$ & [4] \\
\hline Suspected HIT & $\begin{array}{l}\text { Discontinuation of heparin } \\
\text { therapy }\end{array}$ & {$[7,14]$} \\
\hline $\begin{array}{l}\text { Immune thrombocytopenia } \\
\text { (ITP) }\end{array}$ & Corticosteroids & [15] \\
\hline Therapy resistant ITP & Splenectomy & {$[9,10]$} \\
\hline
\end{tabular}

diagnostics, citrate anticoagulated plasma mainly serves for plasmatic coagulation tests. The difference between the anticoagulants is that calcium depletion by EDTA induces irreversible conformational changes of the platelet membrane [17]. Anticoagulant-dependent PTCP is characterized by in vitro platelet aggregation triggered by antibodies, which cross reacts with epitopes of the platelet GPIIb/IIIa receptor $[2,18]$. These binding sites become exposed by EDTA-associated activation and alteration of the platelet membrane [2, 19]; this activity unmasks hidden platelet receptor epitopes of the fibrinogen receptor GPIIb/IIIa (Figure 2). These epitopes are "neoantigens" which bind ubiquitous non-pathogenic autoantibodies resulting in aggregate formation [18, 20-23]. The involvement of the fibrinogen receptor was suggested when platelets from patients with Glanzmann's thrombasthenia did not bind these antibodies [24]

(Modified after Lippi and Plebani [19]; citrate or alternative anticoagulants obviously do not induce this kind of shape change.)

As visualized in Figure 3, the anticoagulant-dependent aggregation of platelets in vitro is time dependent. According to our own experience, the threshold of thrombocytopenia $\left(<150 \times 10_{9} / \mathrm{L}\right)$ is reached after approximately $2 \mathrm{~h}$ of storage when initial values were in the high normal range. The decrease of initially high normal platelet counts leads to lower counts still within the reference range within 

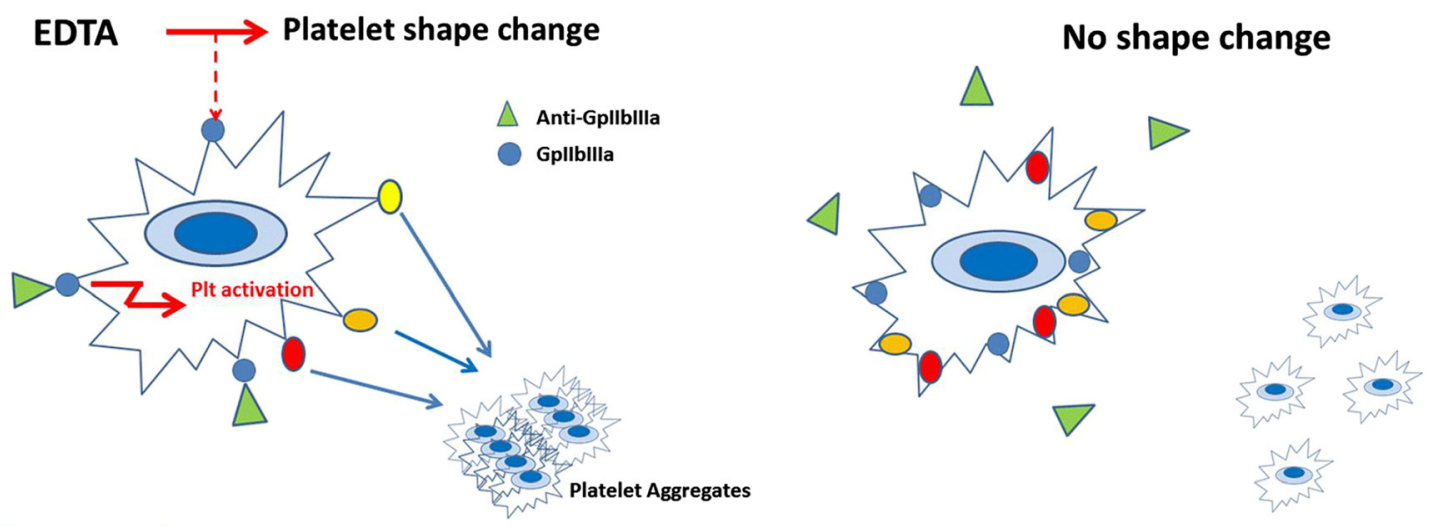

Gmp 140 Gp 55 Thombospondin

Figure 2: Simplified scheme of the mechanism of in vitro platelet aggregation, elicited by EDTA.

the first $2 \mathrm{~h}$ after blood draw. Based on these observations, some investigators believed that anticoagulant-induced aggregation would start after a delay of $2 \mathrm{~h}$. This cannot be confirmed by our own and most of the published observations in PTCP. Temperature might also play a role [25]. Although most autoantibodies involved in PTCP react optimally at temperatures below $20{ }^{\circ} \mathrm{C}$, some autoantibodies, mainly of the IgM class, seem to have their aggregating optimum at $37{ }^{\circ} \mathrm{C}$. This may thus result in spontaneous aggregation in EDTA and in citrateanticoagulated blood samples [26]. The recommendation to measure platelets in freshly drawn or warmed samples does not guarantee correct platelet counts.

Platelet aggregation elicited by EDTA obviously begins immediately with the complexation of extra- and intracellular calcium. The extent of the platelet drop depends on

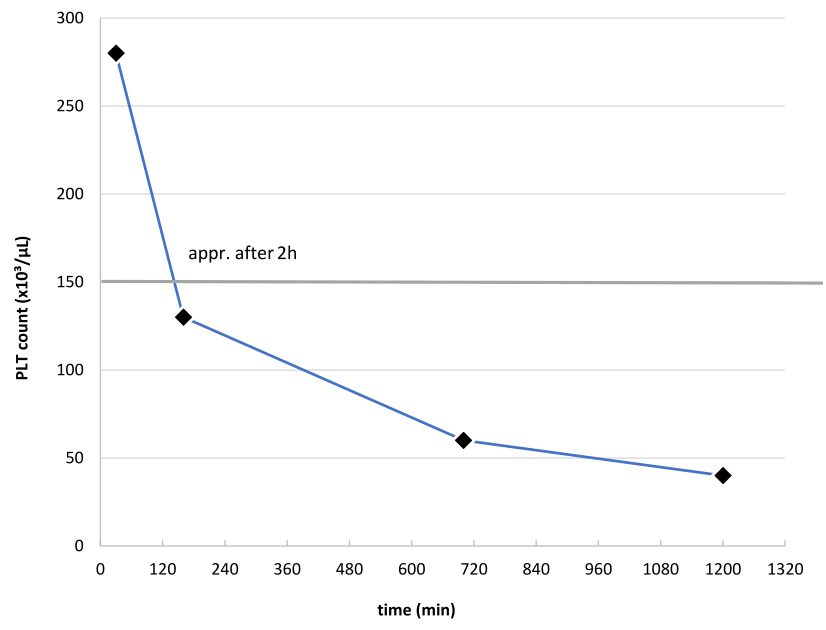

Figure 3: EDTA-induced spontaneous platelet aggregation with consecutive decrease of platelet count with time (representative example from own data). the sample storage time until measurement. This explains the use of the term "pseudo-thrombocytopenia", even though the values are still above counts of $150 \times 10_{9} / \mathrm{L}$. The extent to which the name for this phenomenon justifies the term must remain open.

Because few examples of spontaneous in vitro platelet aggregation were reported from other anticoagulants such as citrate, oxalate and thrombin inhibitors heparin and hirudin, other observers proposed to rename the phenomenon more correctly as "anticoagulant-induced PTCP" [27-29].

\section{Anti-platelet autoantibodies in PTCP}

Bizarro and co-workers [30] used an indirect immunofluorescence assay to detect anti-platelet antibodies in samples of PTCP individuals. Antibodies originated from different immunoglobulin classes, mostly IgG, but also IgM and IgA, and their combinations with IgG were reported by different authors [23, 24, 31].

Casonato and colleagues [18] showed that the "agglutinins" in the serum of healthy and sick PTCP individuals are antibodies of the different immunoglobulin classes covalently binding to the neo-antigen exposed in the presence of EDTA. Therefore, the PTCP phenomenon can be traced back to real aggregation and not to mere agglutination. With their experimental setup, the same authors were able to demonstrate that serum or ammonium plasma precipitates from PTCP individuals led to aggregates in EDTA blood samples from unsuspected subjects. Prior incubation of the serum or plasma precipitates with anti-Ig-antibodies neutralized the aggregating effect, as 
did a specific antibody against the GPIIb/IIIa receptor. The authors could also prove that fibrinogen is not involved in these aggregating effects.

Until now, the origin of the involved nonspecific antibodies remains unclear. Some authors have reported a seasonal fluctuation of PTCP; others have not but were unable to define any reason for their production and sporadic occurrence. Bizarro and Brandalise showed that naturally occurring antibodies react with negativelycharged phospholipids and suggested that they can bind to antigens modified by EDTA on the platelet membrane and would therefore be responsible for PTCP genesis [20, 25].

Abciximab is a well-defined human-mouse chimeric Fab-fragment, binding to the GPIIb/IIIa receptor [32]. It is used as a platelet inhibitor in patients with coronary ischemic syndrome and in percutaneous coronary intervention [33]. The first observation of PTCP associated with abciximab treatment was published in 1984 by Christopolous [34]. A study of abciximab in unstable angina pectoris (CAPTURE) revealed an incidence of PTCP in $2.1 \%$ of the treated patients, whereas only $0.6 \%$ of the placebo treated patients showed platelet aggregates. On the other hand, PTCP was the cause of thrombocytopenia in more than one third $(36.3 \%)$ of patients with coronary heart disease treated with abciximab [33]. Interestingly, abciximabinduced PTCP occurred after the first treatment and was not restricted to EDTA-anticoagulation; in 14 out of 117 patients, platelets aggregated despite citrate anticoagulation [33].It is speculated that the binding of the Fab fragment to the GPIIb/IIIa receptor leads to a change in the conformation of the molecule and thus to the expression of neoantigens against which autoantibodies are then formed. The fact that PTCP occurred after the first treatment suggests that the autoantibodies must already be present [33].

\section{Clinical diagnoses and drug treatment associated with PTCP}

There are numerous casuistic reports on patients' different diagnoses associated with EDTA-dependent spurious platelet counts. As depicted in Table 2, the reported diagnoses make it almost impossible to recognize a certain disease pattern related with PTCP. When all diagnoses are analyzed for disease categories, autoimmune diseases are the most represented, followed by neoplasia. Atherosclerosis-related diagnoses and liver disease are also associated with PTCP at a lower rate [4].In her follow- up study, Bizarro [30] did not confirm that the most common diseases associated with PTCP are autoimmune disorders ( 9 out of 112 individuals). More than half of her study population $(\mathrm{n}=54)$ were healthy individuals undergoing routine health examination. When asked for regular medication, $50.5 \%$ reported the intake of at least one drug, mainly anti-hypertensive medication (diuretics and $\beta$-blockers), tranquilizers, glucose lowering drugs, and oral contraceptive.

There are few reports of PTCP in children suffering from infectious diseases such as mononucleosis [35], dengue fever [36] or HIV [6]. Recently, PTCP was reported in a female patient suffering from acute COVID-19 infection [37]. In these patients one has to differentiate real thrombocytopenia due to the immunological phenomenon of infection, splenic sequestration, or antiretroviral medication from PTCP [38].

\section{Prevalence of PTCP}

The number of patients with PTCP at a given time defines the prevalence within a given population. Prevalence can be also defined as "how many patients currently demonstrate PTCP". This term is often confused with the "incidence", where a phenomenon develops during a defined period [39].

The prevalence of EDTA-induced PTCP obviously depends on the type of study population. In a general outpatient population, this kind of spurious platelet counts ranges between 0.07 and $0.1 \%$ [40]. It is estimated that in random hospitalized patients the PTCP frequency is higher, ranging up to $2 \%$ [41]. In a selected group of thrombocytopenic patients, the reported prevalence ranges between 1.25 and $15.3 \%[2,42]$. This relatively high rate of PTCP in

Table 2: Not all in vitro anticoagulants avoid PTCP (data from the literature).

\begin{tabular}{lllr}
\hline In vitro anticoagulation & Mode of action & PTCP & Reference \\
\hline Sodium citrate & Reversible $\mathrm{Ca}^{++}$binding & Yes & {$[30]$} \\
CPT $^{\mathrm{a}}$ & $\begin{array}{l}\text { (Combined effects) } \\
\text { Heparin }\end{array}$ & No & {$[45]$} \\
& Anti-thrombin & Yes & {$[46]$} \\
Hirudin & amplification & & \\
Ammonium oxalate & $\begin{array}{l}\text { Thrombin inhibition } \\
\text { Sodium fluoride }\end{array}$ & Yes & {$[47]$} \\
Magnesium sulfate & $\mathrm{Ca}^{++}$-chelator & Yes & {$[28,30]$} \\
& $\begin{array}{l}\text { Inhibition of plt } \\
\text { Hydroxy-ethyl- }\end{array} \quad$ activation & No & {$[48]$} \\
$\quad$ theophylline & Inhibition of plt. & No & {$[49]$} \\
\hline
\end{tabular}

${ }^{a}$ Trisodium citrate, pyridoxal-5'phosphate and Tris [44, 45]. 
thrombocytopenic patients indicates a need for primary diagnostics aimed at detection of anticoagulant-dependent in vitro platelet aggregation. Our group evaluated the prevalence of PTCP in three different unpublished studies [43] which are in good accordance with the above cited literature.

\section{Detection, confirmation, or exclusion of PTCP}

The routine hematological laboratory might overlook EDTA-PTCP with the consequence that the treating physician receives incorrect platelet counts. If there is no evidence that the patient has a bleeding tendency (e.g., sudden bleeding events, unexplainable hematoma, prolonged bleeding), pseudo-thrombocytopenia should be excluded before assuming actual thrombocytopenia. Longitudinal monitoring of platelet counts (Figure 4) might facilitate accurate diagnosis of PTCP. As far as the technicians have access to the longitudinal display of laboratory data, they should perform a plausibility check for strongly fluctuating platelet counts and eventually suspect PTCP before reporting the results. Such a case is depicted in Figure 3; the platelet counts in EDTA-anticoagulated blood fluctuate between 28 and $137 \times 10_{9} / \mathrm{L}$ over a follow-up of approximately five years. In citrated samples, measured in parallel, the platelet counts are in the (lower) normal range (142 and $\left.169 \times 10_{9} / \mathrm{L}\right)$.

The blue bars indicate the platelet counts measured in EDTA-anticoagulated blood; the orange bars represent platelet counts measured in citrate-anticoagulated blood in parallel.

Platelet counts within the normal range do not exclude EDTA-induced aggregate formation. This might be the case

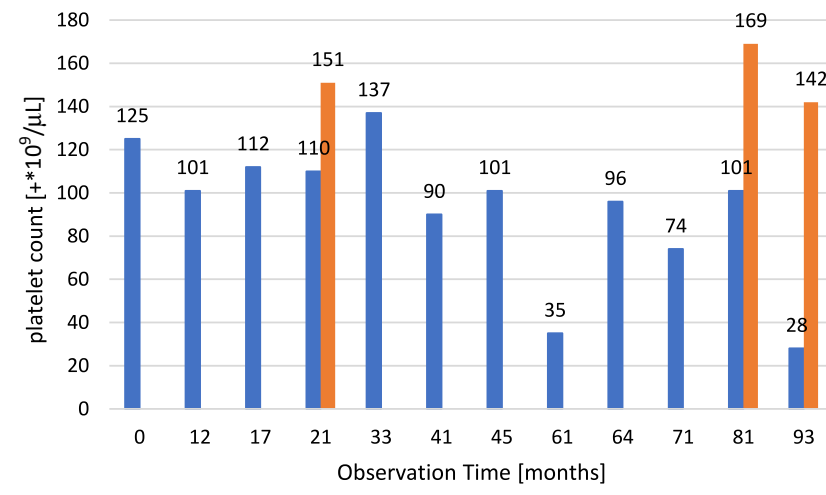

Figure 4: Longitudinal course of EDTA-PTCP over more than 7 years in a 69 years old female patient. if the EDTA-anticoagulated sample with initial platelet counts in the higher portion of the normal range is measured within a short time after blood collection. Because of the time dependency of aggregate formation, the platelet count is not yet in the thrombocytopenic range, although the analyzer flag and the platelet distribution curve indicate platelet aggregates.

This situation explains that samples from PTCP individuals might have "normal" but not accurate platelet counts if measured shortly after sampling. Therefore, the term "pseudo-thrombocytopenia" excludes individuals with EDTA-dependent platelet aggregation but still with normal platelet counts, who probably will be thrombocytopenic when measured again later. The frequency of this event prompted some authors to inform other physicians of the possibility to measure spurious platelet counts when anticoagulated with EDTA. The available literature advises different approaches to confirm or to exclude anticoagulant-dependent PTCP. The simplest but not very reliable approach is to give attention to respective analyzer flags such as "Aggregates?", "Platelet Clumps" or "Giant Platelets". Most of the routine hematology analyzers display flags when measuring blood samples suspected to contain platelet aggregates. To our knowledge, there are only few systemic investigations on the reliability of flagging PTCP samples. This prompted us to perform such still ongoing studies; in our experience the automated hematological analyzer Sysmex XE did not flag all samples with platelet aggregates by the respective warning "Platelet aggregation?” Based on our investigations, sensitivity was calculated at $33.2 \%$, specificity at $97.3 \%$, and efficiency at $96.7 \%$ [43].

The areas framed in red (Figure 5) point to changes that indicate the presence of platelet aggregates: The platelet distribution curve is flattened and serrated like a saw blade, thus leading to the flag "PLT Aggregates"; the immature cell information (IMI)-channel indicates aggregates that present themselves as immature large cells flagged as "Atypical Lymphos?"

The analysis of the platelet distribution curve and the IMI histogram can give additional information with higher sensitivity (83.3\%), but lower specificity (63.3\%) [43]. It is advisable to analyze histograms of thrombocytopenic patients carefully during technical validation, especially if information of previous thrombocytopenia is missing.

As far as we know, there are few published controlled studies [44] concerning the reliability of other types of hematology analyzers for flagging PTCP. However, in a case study from the Netherlands, the authors stated that PTCP samples do not necessarily elicit flagging independent from the type of cell counter [8]. 


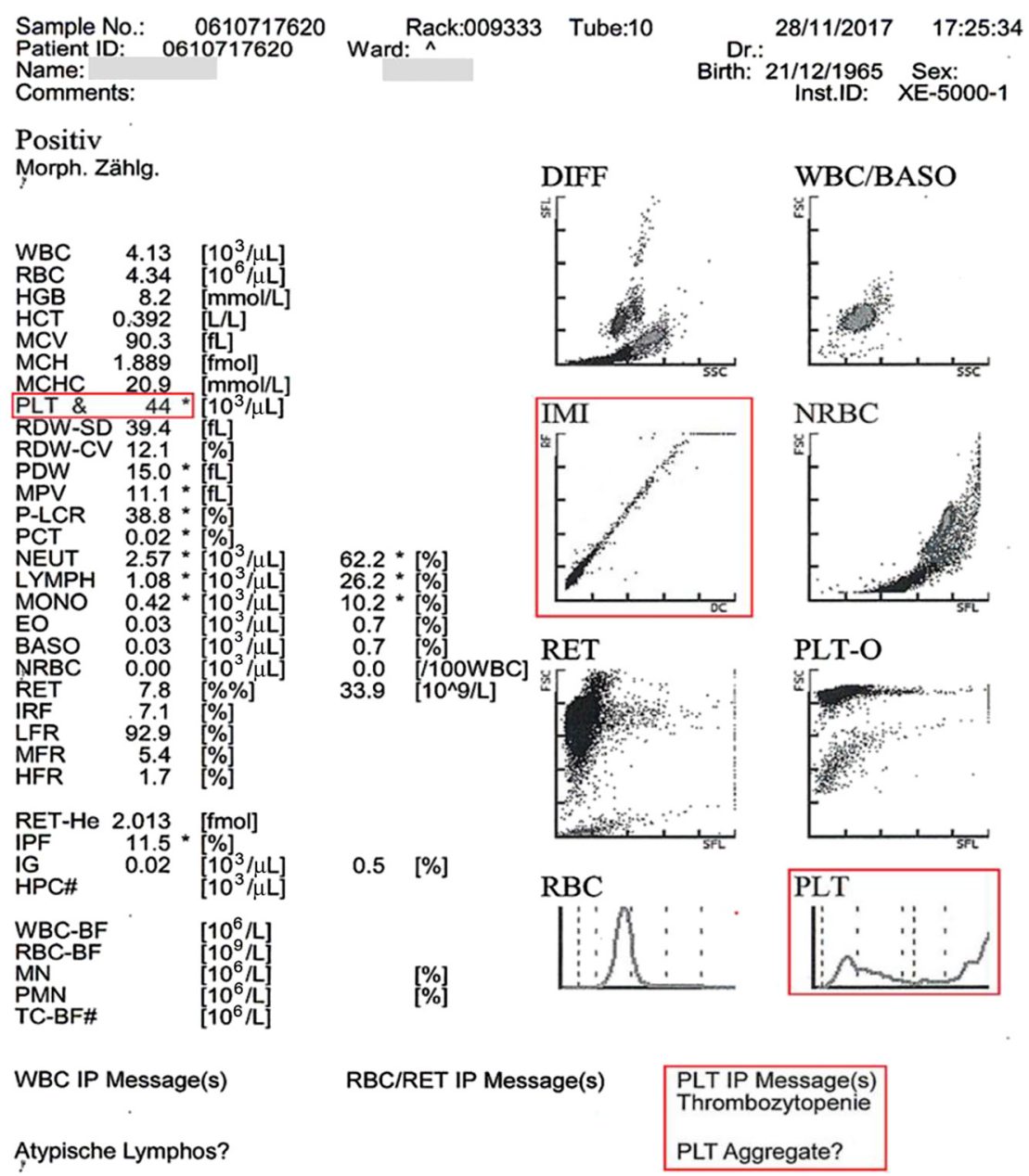

Figure 5: Typical histogram and result printout (Sysmex XE 5000) in patient PTCP with thrombocytopenia indicated by the platelet count and the flag "Thrombocytopenia".

\section{Prevention of anti-coagulant dependent pseudo- thrombocytopenia}

Many studies support that EDTA-related conformational changes are the prerequisite for spontaneous platelet aggregation. Alternative anticoagulants (Table 2) can, but do not have to, prevent such an undesirable effect.

The most recommended alternative is sodium citrate [51], although PTCP was also reported under anticoagulation with citrate [28]. Lippi et al. proposed the use tri-sodium citrate supplemented with pyridoxal 5'-phosphate and neutralized by adding Tris-buffer [44]. This kind of anticoagulation is reported to not affect the cell size.

Sodium citrate anticoagulation is not activating platelets, changing their shape [52], or unmasking neoantigens on the fibrinogen receptor [30]. The mechanism of clump formation by citrate with consecutive lower platelet count seems to be different from that of EDTA. During longer storage under agitation, citrated samples may release some amounts of non-covalently bound calcium, thus eliciting some platelet clump formation (unpublished observation). This might also explain that the platelet distribution curve occasionally shows a discrete saw toothlike course in citrated blood (Figure 6).

The blood was drawn one after the other at the same time and directly transferred to the laboratory. The analyses were performed with a Sysmex XN 2000 hematology analyzer. The distribution curve of the citrateanticoagulated sample (lower left figure) has a discrete saw tooth-like course, a hint for platelet clumps. The inspection of the respective smear revealed clumps in the smear edges.

Nevertheless, PTCP is an exceedingly rare event in citrated blood; such events have mainly been communicated as case reports, although Bizarro also found 15 cases in a collective of 93 PTCP cases triggered by EDTA, which also showed aggregates in blood anticoagulated with citrate [25]. PTCP was also found in citrateanticoagulated samples in $4.1 \%$ of patients treated with abciximab, [33]. 


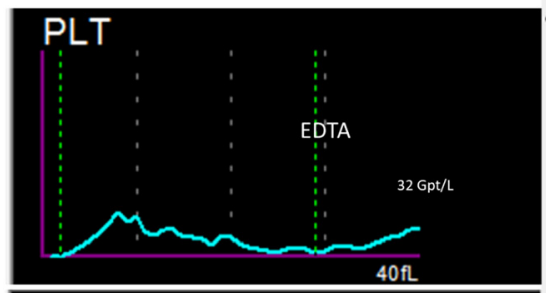

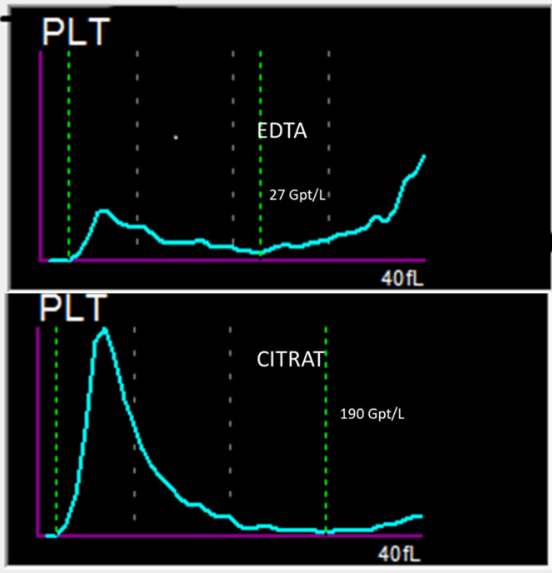

76 yrs old male (0287436)
Figure 6: Platelet counts and screen shots of distribution curves from a 66-year-old female (04027712) and a 76-year-old male (0287436) in dependence of the anti-coagulant.
PTCP was also demonstrated in heparin- or even hirudin-anticoagulated blood samples; both anticoagulants with shortcomings for the hematological complete blood cell count $(\mathrm{CBC})$ routine: Heparin is disadvantageous because of poor staining properties and hirudin is ultimately too expensive.

In 1909, Anton Fonio anticoagulated capillary blood for microscopic platelet counting with a $14 \%$ magnesium sulfate solution [53]. With the development of automated cell counters, EDTA became the standard anticoagulant for estimating cell numbers in the hematological diagnostic routine. The anticoagulant effect of magnesium sulfate was finally no longer mentioned in the literature. Japanese hematologists later rediscovered it in order to prevent EDTA-dependent PTCP [54]. Unfortunately, their positive experience with this alternative anticoagulant was published in Japanese and remained therefore unnoticed until 2013, when a multicentre PTCP-study group reported that magnesium sulfate effectively avoids in vitro platelet aggregation in individuals with EDTA-associated PTCP [49]. This also applies for abciximab-induced aggregate formation leading to false low platelet counts. As shown in Figure 7, blood collection in magnesium supplemented tubes effectively prevents in vitro aggregation in a patient under abciximab treatment.

(Kindly provided to the author by Dr. Michael Steiner, Rostock).

Sodium fluoride, another calcium chelator and coagulant normally used for inhibition of in vitro glycolysis, obviously prevents in vitro aggregation in EDTA-induced PTCP when used in high doses [48]. An interesting approach for avoiding PTCP was published by Sakurai et al. [55] and confirmed by a case report from Korea [56]; supplementation of aminoglycosides to the EDTA

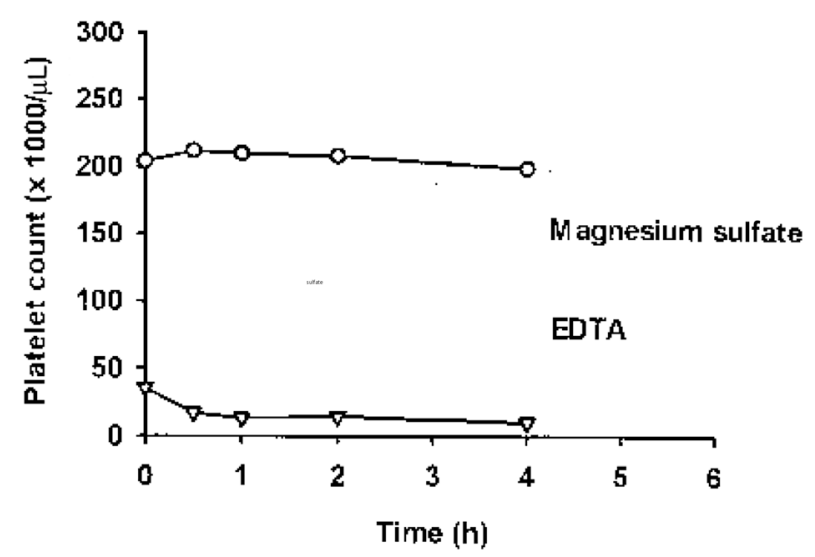

Figure 7: The effect of magnesium sulfate as in vitro anti-coagulant in a patient with Abciximab (Rheo-ProR)-induced PTCP.

venepuncture tube prevented the anti-coagulant related aggregate formation in vitro. The same authors also reported that the addition of the antibiotic to the blood sample resulted in a dissociative effect on the platelet aggregates, leading to higher platelet counts [57]; this does not mean that the real platelet count is measured after this kind of aggregate dissociation. Lombart and colleagues were able to demonstrate the effect of aminoglycosides to avoid EDTA-associated PTCP, but could not confirm the dissociative effect of adding gentamycin to the blood sample [8].

The respective mode of aminoglycoside action on aggregate formation is still speculative [58]. The higher $\mathrm{pH}$ as measured in supplemented probes or conformational changes of the platelet membrane, especially of the GIIb/ IIIa receptor molecule, might play a role. It is conceivable but not yet investigated that other ingredient of the antibiotic drug preparations, such as stabilizers, are responsible for the effects. 
One potential hypothesis is that the patients were treated earlier with aminoglycosides and developed antibodies against the antibiotics, cross-reacting with platelet epitopes unmasked by EDTA exposure. It remains unlikely that addition of aminoglycosides to the PTCP-sample might neutralize those antibodies [55].

Recently, Bao et al. [59] reported a new technology to measure higher platelet counts in EDTA-PTCP based on fluorescence cell analysis (SF-Cube technology), which may be suitable to solve the associated clinical problems. Platelet aggregates dissociate effectively by this technology and platelets are more correctly counted in the CDR (CBC+DIFF+RET) mode without pre-analytical management [60]. These data are not yet confirmed by others. It remains to be seen, however, whether this method can be used to determine the correct platelet count in all cases of thrombocytopenia induced by anticoagulants. Only then PTCP would no longer be a challenge for the laboratory.

\section{Summary, conclusions, and outlook}

After the recommendation of EDTA as in vitro anticoagulant more than 50 years ago, clinical pathologists and clinicians regularly reported spurious low platelet counts in small numbers of their patients. This phenomenon, termed pseudo-thrombocytopenia (PTCP), became a challenge for both the laboratory specialist and the treating physician. If not recognized by the responsible laboratory doctor or technician, the report of false low platelet counts might result in misleading clinical diagnoses or incorrect therapeutic decisions.

The search for predicting factors such as sex, age, medical history, or regular medication was futile. What is striking, however, is that the patients do not have a history of bleeding or recurrent hematoma despite the extremely low platelet counts.

To avoid anticoagulant-induced pseudo-thrombocytopenia, mainly associated with EDTA, is to use either citrate or magnesium sulfate as anticoagulant. Another helpful possibility is to perform the platelet count as early as possible after blood sampling, but this is not suitable for routine use.

Nevertheless, detection, confirmation, or exclusion of PTCP in the daily routine analysis is in the responsibility of the laboratory. The reliability of automated hematologic analyzers is unsatisfying. Thrombocytopenic samples therefore require full attention of the laboratory personnel for checking the warning flags of the hematology analyzer and the histogram for deviations from normal. In pseudothrombocytopenia, the platelet distribution curve often shows a saw tooth-like course. If available, a longitudinal check of preliminary platelet values might be helpful as pseudo-thrombocytopenia shows marked fluctuations in contrast to real thrombocytopenia. In patients with known PTCP, a venepuncture system with an alternative anticoagulant should be used in advance of measuring platelet counts. According to the current state of knowledge, in vitro platelet aggregates have been reported, albeit rarely, for all alternative anticoagulants except for magnesium sulfate. The supplementation of anticoagulated samples with aminoglycosides prior to blood sampling is controversially discussed and ultimately not suitable for routine use.

In the future, the use of anti-coagulants that do not induce in vitro platelet agglutination or the development of more sophisticated algorithms or technologies for recognition of platelet aggregates are necessary.

Research funding: None declared.

Author contributions: All authors have accepted responsibility for the entire content of this manuscript and approved its submission.

Competing interests: Authors state no conflict of interest.

\section{References}

1. Proescher F. Anti-coagulant properties of ethylene bisiminodiacetic acid. Proc Soc Exp Biol Med 1951;76:619-20.

2. Banfi G, Salvagno GL, Lippi G. The role of ethylenediamine tetraacetic acid (EDTA) as in vitro anticoagulant for diagnostic purposes. Clin Chem Lab Med 2007;45:565-7.

3. Gowland E, Kay HE, Spillman JC, Williamson JR. Agglutination of platelets by a serum factor in the presence of EDTA. J Clin Pathol 1969;22:460-4.

4. Berkman N, Michaeli Y, Or R, Eldor A. EDTA-dependent pseudothrombocytopenia: a clinical study of 18 patients and a review of the literature. Am J Hematol 1991;36:195-201.

5. Kohlschein P, Bänsch D, Dreißiger K, Schuff-Werner P. Exclusion of thrombocytopenia as a contraindication for invasive radiofrequency ablation in a patient with paroxysmal atrial fibrillation by using magnesium anticoagulation instead of EDTA: another case of anticoagulant-induced pseudothrombocytopenia. Heart Surg Forum 2015;18:E090-2.

6. Wong VK, Robertson R, Nagaoka G, Ong E, Petz L, Stiehm ER. Pseudothrombocytopenia in a child with the acquired immunodeficiency syndrome. West J Med 1992;157:668-70. 1475958.

7. Zimrin AB, Warkentin TE. Transient pseudothrombocytopenia associated with immune heparin-induced thrombocytopenia complicated by pulmonary embolism. Thromb Haemostasis 2013; 109:971-3.

8. Lombarts AJ, Zijlstra JJ, Peters RH, Thomasson CG. Accurate platelet counting in an insidious case of pseudothrombocytopenia. Clin Chem Lab Med 1999;37:1063-6. 
9. Nilsson T, Norberg B. Thrombocytopenia and pseudothrombocytopenia: a clinical and laboratory problem. Scand J Haematol 1986;37:341-6.

10. Yamada EJ, Souto AFP, Souza EdEO de, Nunes CA, Dias CP. Pseudothrombocytopenia in a patient undergoing splenectomy of an accessory spleen. Case report. Rev Bras Anestesiol 2008; 58:488-91, 485-8.

11. Wilkes NJ, Smith NA, Mallett SV. Anticoagulant-induced pseudothrombocytopenia in a patient presenting for coronary artery bypass grafting. Br J Anaesth 2000;84:640-2.

12. Kocum TH, Katircibasi TM, Sezgin AT, Atalay H. An unusual cause of mismanagement in an acute myocardial infarction case: pseudothrombocytopenia. AJEM (Am J Emerg Med) 2008;26: 740.e1-740.e2.

13. Albersen A, Porcelijn L, Schilders J, Zuetenhorst H, Njo T, Hamberg P. Sunitinib-associated pseudothrombocytopenia induced by IgM antibody. Platelets 2013;24:566-70.

14. Martin-Toutain I, Settegrana C, Ankri A. High levels of heparinplatelet factor 4 antibodies in patients with pseudothrombocytopenia: risk of misdiagnosis. J Thromb Haemostasis 2009;7:1416-8.

15. Le Zhong, Chadha J, Ameri A. A curious case of pseudothrombocytopenia due to in vitro agglutination. Case Rep Hematol 2020;2020:6236350. https://doi.org/10.1155/2020/ 6236350 .

16. Von Meyer A, Cadamuro J, Streichert T, Gurr E, Fiedler GM, Leichtle A, et al. Standard operating procedure for peripheral venous blood sampling. LaboratoriumsMedizin 2017;41:333-40.

17. White JG. Effects of ethylenediamine tetracetic acid (EDTA) on platelet structure. Scand J Haematol 1968;5:241-54.

18. Casonato A, Bertomoro A, Pontara E, Dannhauser D, Lazzaro AR, Girolami A. EDTA dependent pseudothrombocytopenia caused by antibodies against the cytoadhesive receptor of platelet gpIIB-IIIA. J Clin Pathol 1994;47:625-30.

19. Lippi G, Plebani M. EDTA-dependent pseudothrombocytopenia: further insights and recommendations for prevention of a clinically threatening artifact. Clin Chem Lab Med 2012;50:1281-5.

20. Bizzaro N, Brandalise M. EDTA-dependent pseudothrombocytopenia. Association with antiplatelet and antiphospholipid antibodies. Am J Clin Pathol 1995;103:103-07.

21. Mant MJ, Doery JCG, Gauldie J, Sims H. Pseudothrombocytopenia due to platelet aggregation and degranulation in blood collected in EDTA. Scand J Haematol 1975;15:161-70.

22. Pegels JG, Bruynes EC, Engelfriet CP, Von dem Borne AE. Pseudothrombocytopenia: an immunologic study on platelet antibodies dependent on ethylene diamine tetra-acetate. Blood 1982;59:157-61.

23. van Vliet HH, Kappers-Klunne MC, Abels J. Pseudothrombocytopenia: a cold autoantibody against platelet glycoprotein GP Ilb. Br J Haematol 1986;62:501-11.

24. Veenhoven WA, van der Schans GS, Huiges W, MettingScherphuis HE, Halie MR, Nieweg HO. Pseudothrombocytopenia due to agglutinins. Am J Clin Pathol 1979;72:1005-08.

25. Bizzaro N. Pseudothrombocytopenia and platelet antigens. Clin Appl Thromb Hemost 1999;5:141. 10725998.

26. van der Meer W, Allebes W, Simon A, Van Berkel Y, De Keijzer $\mathrm{MH}$. Pseudothrombocytopenia: a report of a new method to count platelets in a patient with EDTA- and temperature- independent antibodies of the IgM type. Eur J Haematol 2002; 69:243-7.

27. Rosenkranz B, Wisser H, Bode JC. Pseudothrombopenie-eine störung der Thrombozytenzahlbestimmung. Dtsch Med Wochenschr 1985;110:492-5.

28. Schrezenmeier H, Müller H, Gunsilius E, Heimpel H, Seifried E. Anticoagulant-induced pseudothrombocytopenia and pseudoleucocytosis. Thromb Haemostasis 1995;73:506-13.

29. Kovacs F, Varga M, Pataki Z, Rigo E. Pseudothrombocytopenia with multiple anticoagulant sample collection tubes. Intervent Med Appl Sci 2016;8:181-3.

30. Bizzaro N. EDTA-dependent pseudothrombocytopenia: a clinical and epidemiological study of 112 cases, with 10-year follow-up. Am J Hematol 1995;50:103-9.

31. Payne BA. EDTA-induced pseudothrombocytopenia. Recognizing a laboratory artifact. Postgrad Med 1985;77:75-6.

32. Coller BS. A new murine monoclonal antibody reports an activation-dependent change in the conformation and/or microenvironment of the platelet glycoprotein IIb/IIla complex. J Clin Invest 1985;76:101-08.

33. Sane DC, Damaraju LV, Topol EJ, Cabot CF, Mascelli MA, Harrington RA, et al. Occurrence and clinical significance of pseudothrombocytopenia during abciximab therapy. J Am Coll Cardiol 2000;36:75-83.

34. Christopoulos CG, Machin SJ. A new type of pseudothrombocytopenia: EDTA-mediated agglutination of platelets bearing Fab fragments of a chimaeric antibody. $\mathrm{Br}$ J Haematol 1994;87:650-2.

35. Hsieh AT, Chao TY, Chen YC. Pseudothrombocytopenia associated with infectious mononucleosis. Arch Pathol Lab Med 2003;127:e17-8.

36. Vaidya $P$, Venkataraman R. Pseudothrombocytopenia in a child with dengue. Indian J Pediatr 2014;81:1395-6.

37. Li H, Wang B, Ning L, Luo Y, Xiang S. Transient appearance of EDTA dependent pseudothrombocytopenia in a patient with 2019 novel coronavirus pneumonia. Platelets 2020:1-2. https://doi.org/10. 1080/09537104.2020.1760231.

38. Ellaurie M, Burns ER, Bernstein LJ, Shah K, Rubinstein A. Thrombocytopenia and human immunodeficiency virus in children. Pediatrics 1988;82:905-8.

39. Ramfjord SP. Indices for prevalence and incidence of periodontal disease. J Peridontol 1959;30:51-9.

40. Bartels PCM, Schoorl M, Lombarts AJPF. Screening for EDTA-dependent deviations in platelet counts and abnormalities in platelet distribution histograms in pseudothrombocytopenia. Scand J Clin Lab Invest 1997;57:629-36.

41. Vicari A, Banfi G, Bonini PA. EDTA-dependent pseudothrombocytopaenia: a 12-month epidemiological study. Scand J Clin Lab Invest 1988;48:537-42.

42. Silvestri F, Virgolini L, Savignano C, Zaja F, Velisig M, Baccarani $M$. Incidence and diagnosis of EDTA-dependent pseudothrombocytopenia in a consecutive outpatient population referred for isolated thrombocytopenia. Vox Sang 1995;68:35-9.

43. Mansour J, Mannuß S, Gropp A, Scharfenberg K, Schuff-Werner P. Prevalence of EDTA-anticoagulation associated platelet agglutination in a large outpatient laboratory: specificity and sensitivity of Sysmex XE 5000 flagging for platelet aggregates. ISLH 2019 abstracts. Int J Lab Hematol 2019:108. https://doi.org/ 10.1111/ijlh.13105. 
44. Lippi U, Schinella M, Nicoli M, Modena N, Lippi G. EDTA-induced platelet aggregation can be avoided by a new anticoagulant also suitable for automated complete blood count. Haematologica 1990;75:38-41. 2110927.

45. Lippi G, Guidi G, Nicoli M. Platelet count in EDTA-dependent pseudothrombocytopenia. Eur J Haematol 1996;56:112-13.

46. Savage RA. Pseudoleukocytosis due to EDTA-induced platelet clumping. Am J Clin Pathol 1984;81:317-22.

47. Robier C, Neubauer M, Sternad H, Rainer F. Hirudin-induced pseudothrombocytopenia in a patient with EDTA-dependent platelet aggregation: report of a new laboratory artefact. Int J Lit Humanit 2010;32:452-53.

48. Kabutomori O, Iwatani Y. "Correct” platelet count in EDTA-dependent pseudothrombocytopenia. Eur J Haematol 1995;55:67-8.

49. Schuff-Werner P, Steiner M, Fenger S, Gross HJ, Bierlich A, Dreissiger K, et al. Effective estimation of correct platelet counts in pseudothrombocytopenia using an alternative anticoagulant based on magnesium salt. Br J Haematol 2013;162:684-92.

50. Ohnuma 0 , Shirata Y, Miyazawa K. Use of theophylline in the investigation of pseudothrombocytopenia induced by edetic acid (EDTA-2K). J Clin Pathol 1988;41:915-17.

51. Lin J, Luo Y, Yao S, Yan M, Li J, Ouyang W, et al. Discovery and correction of spurious low platelet counts due to EDTA-dependent pseudothrombocytopenia. J Clin Lab Anal 2015;29:419-26.

52. Patscheke H. Shape and functional properties of human platelets washed with acid citrate. Haemostasis 1981;10:14-27.

53. Fonio A. Über ein neues Verfahren der Blutplättchenzählung. Dt Ztsch Chir 1912;117:176-94.
54. Nakamoto K, Sugibayashi S, Takahashi A, Terauchi S, Hada A, Munakata $\mathrm{M}$, et al. Platelet count in EDTA-dependent pseudothrombocytopenia-application of $\mathrm{MgSO}_{4}$ as an anticoagulant. Rinsho Byori 1986;34:167-73. 3084831.

55. Sakurai S, Shiojima I, Tanigawa T, Nakahara K. Aminoglycosides prevent and dissociate the aggregation of platelets in patients with EDTA-dependent pseudothrombocytopenia. Br J Haematol 1997;99:817-23.

56. Ahn HL, Jo YI, Choi YS, Lee JY, Lee HW, Kim SR, et al. EDTA-dependent pseudothrombocytopenia confirmed by supplementation of kanamycin; a case report. Korean J Intern Med 2002;17:65-8.

57. Bokaei PB, Grabovsky D, Shehata N, Wang C. Impact of amikacin on pseudothrombocytopenia. Acta Haematol 2017; 137:27-9.

58. Ozcelik F, Arslan E, Serdar MA, Kurt I, Yiginer O, Kayadibi H, et al. A useful method for the detection of ethylenediaminetetraacetic acid- and cold agglutinin-dependent pseudothrombocytopenia. Am J Med Sci 2012;344:357-62.

59. Bao Y, Wang J, Wang A, Bian J, Jin Y. Correction of spurious low platelet counts by optical fluorescence platelet counting of BC-6800 hematology analyzer in EDTA-dependent pseudo thrombocytopenia patients. Transl Cancer Res 2020;9: 166-72.

60. Deng J, Chen Y, Zhang S, Li L, Shi Q, Liu M, et al. Mindray SF-Cube technology: an effective way for correcting platelet count in individuals with EDTA dependent pseudo thrombocytopenia. Clin Chim Acta 2020;502:99-101. 